\title{
Circuit depth relative to a random oracle*
}

\author{
Peter Bro Miltersen \\ Aarhus University, Computer Science Department \\ Ny Munkegade, DK 8000 Aarhus C, Denmark. \\ bromille@daimi.aau.dk
}

August 1991

Keywords: Computational complexity, random oracles, circuit depth.

\section{Introduction}

The study of separation of complexity classes with respect to random oracles was initiated by Bennett and Gill [1] and continued by many authors.

Wilson $[5,6]$ defined relativized circuit depth and constructed various oracles $A$ for which $P^{A} \neq N C^{A}, N C_{k}^{A} \neq N C_{k+\epsilon}^{A}, A C_{k}^{A} \neq A C_{k+\epsilon}^{A}, A C_{k}^{A} \nsubseteq$ $N C_{k+1-\epsilon}^{A}$ and $N C_{k}^{A} \not \subset A C_{k-\epsilon}^{A}$ for all positive rational $k$ and $\epsilon$, thus separating those classes for which no trivial argument shows inclusion. In this note we show that as a consequence of a single lemma, these separations (or improvements of them) hold with respect to a random oracle $A$.

\section{The results}

Let $\Sigma=\{0,1\}$ and let $\log n$ denote $\log _{2} n$. Recall the following definitions by Wilson $[4,5,6]$.

*This research was partially supported by the ESPRIT II Basic Research actions Program the EC under contract No. 3075 (project ALCOM). 
Definition $1 A$ bounded fan-in oracle circuit $C$ is a circuit containing negation gates of indegree 1, and and or gates of indegree 2 as well as of unspecified oracle gates of various indegrees, giving a single boolean output. Given an oracle $A$, i.e. a subset of $\Sigma^{*}, C^{A}$ denotes the circuit, where each oracle gate of indegree $m$ in $C$ has been replaced by a gate computing $\chi_{A}: \Sigma^{m} \rightarrow \Sigma$, where $\chi_{A}(x)$ is 1 if $x \in A$ and 0 otherwise. The depth of an oracle gate with $n$ inputs is $\lceil\log n\rceil$. The size of an oracle gate with $n$ inputs is $n-1$. The boolean gates have size and depth 1 . The size of an oracle circuit is the sum of the sizes of its gates. The depth of a path in the circuit is the sum of the depths of the gates along the path. The depth of the circuit is the depth of its deepest path.

Definition 2 An unbounded fan-in oracle circuit $C$ is defined as in the bounded fan-in case, except that and and or gates of arbitrary indegree are allowed, and each oracle gate is only charged a depth of 1 . The depth of an unbounded fan-in circuit is thus simply the length of its longest path.

Definition 3 DEPTH i.o. $^{A}(d)$ is the class of functions $f$ so that for infinitely many integers $n$ a bounded fan-in oracle curcuit $C_{n}$ with $n$ inputs of depth at most $d$ exists, so that $C_{n}^{A}(x)=f(x)$ for all $x \in \Sigma^{n}$, where $C_{n}^{A}(x)$ denotes the output of $C_{n}^{A}$ when $x$ is given as input.

Let $k$ be a positive rational number. $N C_{k}^{A}$ is the class of functions $f$ for which a logspace-uniform family of polynomial size, $O\left(\operatorname{Iog}^{k} n\right)$-depth bounded fan-in curcuits $C_{n}$ with $n$ inputs exists, so that $C_{n}^{A}(x)=f(x) . A C_{k}^{A}$ is the class of functions $f$ for which a logspace-uniform family of polynomial size, $O\left(\log ^{k} n\right)$-depth unbounded fan-in circuits $C_{n}$ with $n$ inputs exists, so that $C_{n}^{A}(x)=f(x)$.

Let $A$ be an oracle. Let $t_{1}^{n}, \ldots, t_{n}^{n}$ be the $n$ lexicographically first strings of length $\lceil\log n\rceil$. Let $f_{n}^{A}:\{0,1\}^{n} \rightarrow\{0,1\}^{n}$ be the function $f_{n}^{A}(x)=$ $\chi_{A}\left(x t_{1}^{n}\right) \chi_{A}\left(x t_{2}^{n}\right) \cdots \chi_{A}\left(x t_{n}^{n}\right)$.

Lemma 4 Let $n$ and $d$ be positive integers. Let $C$ be a fixed oracle curcuit with $n$ boolean inputs and $n$ boolean outputs containing at most $s=$ $2^{\frac{n}{2}-2 \log d-5}$ oracle gates of indegree exactly $n+\lceil\log n\rceil$ so that no path in $C$ contains more than $d$ oracle gates of indegree exactly $n+\lceil\log n\rceil$ (no restric- 
tions is made on gates of other indegrees). Then, for a random oracle $A$, the probability that $C^{A}$ computes $\left(f_{n}^{A}\right)^{d+1}$, i.e. the composition of $f_{n}^{A}$ with itself $d+1$ times, is at most $2^{-2 \frac{n}{2}}$.

Proof Let us call the oracle gates of indegree $n+\lceil\log n\rceil$ for interesting. We partition the gates of $C$ into $d$ levels $0,1, \ldots, d-1$, such that no path exists from the output of any interesting gate at level $i$ to the input of any interesting gate at level $j$ if $j \leq i$. The idea of the proof is to show that with high probability, $\left(f_{n}^{A}\right)^{i+1}(x)$ is not computed before level $i$. Given an oracle $A$ and a vector $x \in \Sigma^{n}$, let $I_{x}^{A}(i)$ denote the set of strings $y$ for which some string $t$ of length $\lceil\log n\rceil$ exists, so that $y t$ is given as input to some interesting gate at level $i$, when $C^{A}$ is given $x$ as an input. For convenience, let $I_{x}^{A}(d)=\left\{C^{A}(x)\right\}$. $(x)$.

Consider the following procedure for finding an $x$ so that $C^{A}(x) \neq\left(f_{n}^{A}\right)^{d+1}$

1. $L:=\emptyset$.

2. if $\Sigma^{n} \subseteq L$ then abort, we were not successful.

3. select any $x \in \Sigma^{n} \backslash L$.

4. $x_{0}:=x$.

5. for $i:=0$ to $d$ do

6. compute $I_{x}^{A}(i)$ by simulating the necessary parts of the circuit.

7. $L:=L \cup I_{x}^{A}(i) \cup\left\{x_{i}\right\}$.

8. $x_{i+1}:=f_{n}^{A}\left(x_{i}\right)$.

9. if $x_{i+1} \in L$ then goto 2 .

10. od.

11. return $x$.

Let us first observe that the protocol indeed returns an $x$ with the desired property in case it does not abort. This is so, because $x_{d+1}=\left(f_{n}^{A}\right)^{d+1}(x)$, and 
the algorithm makes sure that $x_{d+1} \notin L$ at a time when $I_{x}^{A}(d) \subseteq L$ and by definition $C^{A}(x) \in I_{x}^{A}(d)$. Let us then estimate the probability of abortion. We will first give an upper bound on the probability of leaving the for-loop at line 9. For convenience, let us assume that the membership of a string in $A$ is not determined until the algorithm asks for it. It is easy to see that the protocol makes sure that no bit of the value of $f_{n}^{A}\left(x_{i}\right)$ has been determined previous to line 8 . Hence, all $2^{n}$ values are equally likely. Of these values, $|L|$ causes the algorithm to leave the for-loop in the next line. Hence, each time line 9 is encountered, the probability of leaving the loop is exactly $\frac{|L|}{2^{n}}$. If we assume that $m$ values of $x$ has been tried so far (including the current value), an upper bound of this is $\frac{m(s+d+1)}{2^{n}} \leq \frac{3 d m s}{2^{n}}$. Thus, each time the for-loop is executed, an upper bound of the probability of leaving it prematurely is $(d+1) \frac{3 d m s}{2^{n}} \leq \frac{6 d^{2} m s}{2^{n}}$. Since the algorithm will try different values of $x$ at least until this upper bound is 1 and the above argument applies to all of them, we have that for any positive integer $k$ :

$$
\operatorname{Pr}(\text { abortion }) \leq \prod_{m=1}^{\left\lfloor\frac{2^{n}}{6 d^{2} m s}\right\rfloor} \frac{6 d^{2} m s}{2^{n}} \leq\left(\frac{6 d^{2} k s}{2^{n}}\right)^{k}
$$

Putting $k=\left\lceil 2^{\frac{n}{2}}\right\rceil$, we get:

$$
\operatorname{Pr}(\text { abortion }) \leq 2^{-2 \frac{n}{2}}
$$

Theorem 5 For $\alpha<\frac{1}{2}, P^{A} \nsubseteq D E P T H_{\text {i.o. }}^{A}(\alpha n)$ for a random oracle $A$ with probability 1.

Proof Let $d_{n}=\lfloor\alpha n\rfloor$. The family of functions $g_{n}^{A}=\left(f_{n}^{A}\right)^{d_{n}+1}$ is clearly in $P^{A}$. Fix $n$ and let $C$ be a fixed bounded fan-in oracle circuit of depth $d_{n}$. It is easy to see that the size of $C$ is at most $2^{d_{n}}$, so by the lemma, the probability that $C^{A}$ computes $g_{n}^{A}$ is at most $2^{-2^{\frac{n}{2}}}$. There are at most $2^{2^{d_{n}+o\left(d_{n}\right)}}$ bounded fan-in oracle circuits of depth $d_{n}$, so the probability that some such circuit computes $g_{n}^{A}$ with $A$ as oracle is at most $2^{2^{\alpha n+o(n)}} 2^{-2^{\frac{n}{2}}}$ which is less than $2^{-n}$ for sufficiently large $n$. Thus, for fixed $N$, the probability that for some $n$ greater than $N, g_{n}^{A}$ has $A$-circuits of depth at most $\alpha n$, is at most $\sum_{n=N}^{\infty} 2^{-n}=2^{-N+1}$. The probability that for all $N$, an $n$ greater than $N$ exists, so that $g_{n}^{A}$ has circuits of depth at most $\alpha n$, is thus at most 
$\inf _{N} 2^{-N+1}=0$

The theorem is an improvement of Wilson's result [5] that oracles $A$ exists, so that $P^{A} \neq N C^{A}$. Since every function has unrelativized depth at most $n+o(n)$, the result is optimal, up to a multiplicative constant of $2+\epsilon$. Similar results about circuit size were obtained by Lutz and Schmidt [3] who showed that for small $\alpha$ and a random oracle $A, N P^{A} \nsubseteq S I Z E_{i . o .}^{A}\left(2^{\alpha n}\right)$ and by Kurtz, Mosey and Royer [2], who proved $N P^{A} \nsubseteq c o-N S I Z E_{i . o .}^{A}\left(2^{\alpha n}\right)$.

Theorem 6 For rational $k \geq 0$ and $\epsilon>0, A C_{k}^{A} \nsubseteq N C_{k+1-\epsilon}^{A}$ for random $A$ with probability 1.

Proof Let $d_{n}=\left\lfloor\log ^{k} n\right\rfloor$ and $g_{n}^{A}=\left(f_{n}^{A}\right)^{d_{n}+1} \cdot g_{n}^{A}$ is in $A C_{k}^{A}$. It is sufficient to prove that with probability $1, g_{n}^{A}$ is not computed by a family of bounded fan-in circuits $C_{n}$ of depth $O\left(\log ^{k+1-\epsilon} n\right)$. Fix an $n$ and a circuit $C_{n}$ within this bound. Observe that $C_{n}$ can not contain a path with more than $O\left(\log ^{k-\epsilon} n\right)$ oracle gates of indegree $n+\lceil\log n\rceil$ and that $C_{n}$ satisfies the size bound of the lemma. Thus, the probability that $C_{n}^{A}$ computes $g_{n}^{A}$ is at most $2^{-2 \frac{n}{2}}$. Now proceed as in the previous proof.

It is easy to see from the proof that we actually get the stronger result that there are functions in $A C_{n}^{A}$ which can not be computed in depth $o\left(\log ^{k+1} n\right)$ by bounded fan-in $A$-circuits.

Theorem 7 For rational $k>0$ and $\epsilon>0, N C_{k}^{A} \nsubseteq A C_{k-\epsilon}^{A}$ for random A with probability 1.

Proof The proof is bred upon the idea behind the corresponding oracle construction by Wilson [6]. Let $d_{n}=\left\lfloor\frac{\log ^{k} n}{\log \log n}\right\rfloor, m_{n}=\left\lceil\log ^{2} n\right\rceil$ and let $g_{n}^{A}\left(x_{1} x_{2} \ldots x_{n}\right)=\left(f_{m n}^{A}\right)^{d_{n}+1}\left(x_{1} x_{2} \ldots x_{m_{n}}\right) . g_{n}^{A}$ is in $N C_{k}^{A}$, since we are only charged depth $O(\log \log n)$ for computing $f_{m_{n}}^{A}$. The probability that $g_{n}^{A}$ is computed by a specific circuit of size $O\left(n^{l}\right)$, depth $O\left(\log ^{k-\epsilon} n\right)$, even with unbounded fan-in, is, by the lemma, at most $2^{-2 \frac{m_{n}}{2}} \leq 2^{-n^{\frac{\log n}{2}}}$. Now proceed as in the previous proofs.

The proof actually gives us functions in $N C_{k}^{A}$ which require superpolynomial size to be computed in depth $o\left(\log ^{k} n / \log \log n\right)$ with unbounded fan-in $A$ - 
circuits. This is optimal, since standard techniques provide a simulation of $N C_{k}^{A}$ by polynomial size, depth $O\left(\log ^{k} n / \log \log n\right)$, unbounded fan-in $A$ circuits.

Corollary 8 For rational $k \geq 0$ and $\epsilon>0, N C_{k}^{A} \neq N C_{k+\epsilon}^{A}$ and $A C_{k}^{A} \neq$ $A C_{k+\epsilon}^{A}$ for random A with probability 1.

\section{References}

[1] C.H. Bennett and J. Gill: Relative to a random oracle A, $P^{A} \neq N P^{A} \neq$ co $-N P^{A}$ with probability 1, SIAM J. Comput. 10 (1981) 96-113.

[2] S. Kurtz, S. Mahaney and J. Royer, Average dependence and random oracles, Tech. Rept. SU-CIS-91-03, School of Computer and Information Science, Syracuse University, January 1991.

[3] J.H. Lutz and W.J. Schmidt, Circuit size relative to pseudorandom oracles, in: Proc. 5th Structure in Complexity Theory Conference (IEEE Press, 1990) 268-286. Errata in: Proc. 6th Structure in Complexity Theory Conference (IEEE Press, 1991) 392.

[4] C.B. Wilson, Relativized circuit complexity, J. Comput. System Sci. 31 (1985) 169-181.

[5] C.B. Wilson, Relativized NC, Math. Systems Theory 20 (1987) 13-29.

[6] C.B. Wilson, On the decomposability of $N C$ and $A C$, SIAM J. Comput. 19 (1990) 384-396. 